\title{
An alternative import pathway of AIF to the mitochondria
}

\author{
SHU-FEN CHIANG ${ }^{1}$, CHIH-YANG HUANG ${ }^{1}$, TZE-YI LIN ${ }^{3,4}$, \\ SHIOW-HER $\mathrm{CHIOU}^{1}$ and KUAN-CHIH CHOW ${ }^{2}$
}

\author{
${ }^{1}$ Graduate Institute of Microbiology and Public Health; ${ }^{2}$ Graduate Institute of Biomedical Sciences, \\ National Chung Hsing University; ${ }^{3}$ Department of Pathology, College of Medicine, China Medical \\ University Hospital; ${ }^{4}$ College of Medicine, China Medical University, Taichung, Taiwan, R.O.C.
}

Received September 15, 2011; Accepted November 7, 2011

DOI: $10.3892 / \mathrm{ijmm} .2011 .849$

\begin{abstract}
In eukaryotic cells, transport of the newly synthesized proteins and phospholipids to the appropriate subcellular target compartments is essential for maintaining organelle morphology and cell survival. In animal cells, mitochondria are major organelles containing DNA genome that encodes only for a small fraction of their proteins, which are required for the organelle function. Most mitochondrial proteins are encoded by the nuclear genes and imported to the mitochondria following protein synthesis. Apoptosis-inducing factor (AIF), an essential FAD-dependent NADH oxidase for the oxidative phosphorylation, is located in the intermembranous space and contains mitochondrial localization signals. However, the import mechanism of AIF to the mitochondria is not yet studied. Using sucrose gradient ultracentrifugation and immunoblotting, AIF was detected in fractions of the endoplasmic reticulum, mitochondria-associated membranes (MAM) and mitochondria, and AIF from these fractions was resistant to trypsin in the absence of digitonin, suggesting that AIF could be protected by phospholipids. Knockdown of
\end{abstract}

Correspondence to: Dr Kuan-Chih Chow, Graduate Institute of Biomedical Sciences, National Chung Hsing University, Taichung, Taiwan, R.O.C

E-mail: kcchow@dragon.nchu.edu.tw

Dr Shiow-Her Chiou, Graduate Institute of Microbiology and Public Health, Taichung, Taiwan, R.O.C.

E-mail: shchiou@dragon.nchu.edu.tw

Abbreviations: AIF, apoptosis-inducing factor; ATAD3A, ATPase family AAA domain containing 3A; DRP1, dynamin-related protein 1; ER, endoplasmic reticulum; MAM, mitochondria-associated membrane; Mfn-2, mitofusin-2; MLS, mitochondrial localization signal; MOM, mitochondrial outer membrane; PC, phosphatidylcholine; PE, phosphatidylethanolamine; PM, post-mitochondrial; PS, phosphatidylserine; TIM, translocase of mitochondrial inner membrane; TOM, translocase of mitochondrial outer membrane

Key words: ATPase family AAA domain containing 3A, dynaminrelated protein 1, endoplasmic reticulum, mitofusin-2, mitochondria, mitochondria-associated membrane, programmed cell death protein 8 , transport vesicles dynamin-related protein $1\left(\mathrm{DRP}^{\mathrm{kd}}\right)$ expression reduced AIF levels in the mitochondria, but increased AIF concentrations in the MAM. Knockdown of mitofusin-2 $\left(\mathrm{Mfn}-2^{\mathrm{kd}}\right)$ or ATPase family AAA domain containing $3 \mathrm{~A}\left(\mathrm{ATAD} \mathrm{A}^{\mathrm{kd}}\right.$ ) expression, however, reduced AIF levels in the mitochondria and increased the number of transport vesicles that contained AIF in the cytosol, indicating that ATAD3A and Mfn-2 were respectively essential for the import and fusion of transport vesicles into the mitochondria. Here we show that AIF is imported from the endoplasmic reticulum to the mitochondria via mitochondriaassociated membranes and transport vesicles.

\section{Introduction}

Mitochondria are major organelles that have their own DNA genomes. However, their genomes encode only for 13 proteins that are part of the oxidative phosphorylation. Most of the estimated 1,500 human proteins, which are essential for the mitochondrial function, are nuclear encoded. Some of these proteins are synthesized in the cytosol as precursor or co-translational proteins, and imported to mitochondria via the mitochondrial localization signal (MLS) and translocase of the mitochondrial outer membrane (TOM) complex $(1,2)$. The precursor proteins are guided by chaperones to receptor of TOM complex and imported into mitochondria $(3,4)$. Co-translational proteins, on the other hand, are synthesized on mitochondria-associated ribosomes, forming a nascent polypeptide-associated complex (NAC) on the surface of the mitochondria and directly imported into the mitochondria $(1,5)$.

Apoptosis-inducing factor (AIF), a FAD-dependent NADH oxidase, has been shown to play an important role in the oxidative phosphorylation of mitochondrial complex I and III (6). The full-length AIF contains 2 potential MLS at the N-terminus, and 2 putative nuclear localization signals (NLS) in the middle of the protein (7). Interestingly, AIF is located in the intermembranous space (IMS). Presence of the free AIF in the cytoplasm, however, is hazardous to cells. Following genotoxic challenges, AIF is released from the mitochondria, and further translocated to the nucleus to trigger DNA fragmentation and initiation of apoptosis (7-9). To prevent cells from unprogrammed apoptosis, the newly synthesized AIF should be protected by chaperones or be rapidly concealed before transporting to the mitochondria. 
However, the mechanism of importing AIF to mitochondria has not been studied.

Using antibodies specific to the ATPase family AAA domain containing 3A (ATAD3A) to react with mitochondriaassociated membrane (MAM) and light membrane fractions of sucrose gradient ultracentrifugation before immunoblotting, proteins, such as dynamin-related protein 1 (DRP1), eukaryotic elongation factor 2 (eEF2), mitofusin-2 (Mfn-2), optic atrophy protein 1 (OPA1), and AIF were all co-precipitated (10). Antibodies specific to AIF, however, did not precipitate AIF or the above-mentioned proteins, indicating that AIF was shielded away from interacting with antibodies, possibly by phospholipids. Interestingly, all these proteins were detected on the endoplasmic reticulum (ER), MAM and mitochondria (10). DRP1 and Mfn-2 have been clearly shown to be involved in mitochondrial shaping. Mfn-2, in addition to its essential role in mitochondrial fusion, is imperative for maintaining close contact between the ER (possibly MAM) and mitochondria (11-15). DRP1, a GTPase, is not only required for mitochondrial fission, but also mediates vesicle budding from Golgi in plants (16). Moreover, when studying expression of DRP1, Mfn-2 and ATAD3A in lung cancer cells, we found that silencing of DRP1 increased bulging of MAM (10). Silencing of ATAD3A increased mitochondrial fragmentation and the number of vesicles around dilated MAM. Knockdown of Mfn-2, however, increased the number of vesicles around the mitochondria in addition to mitochondrial fragmentation. Taken together, we hypothesized that there could be an alternative import pathway for some of the mitochondrial proteins. At least 3 proteins, i.e., DRP1, ATAD3A and Mfn-2, are required for this protein trafficking pathway (10). In this report, our data show that some of the mitochondrial proteins, e.g., AIF, are synthesized in the ER and packaged, en route through mitochondria-associated membranes, into transport vesicles and imported to the mitochondria before fusion with the mitochondrial outer membrane.

\section{Materials and methods}

Cell culture. Three human cancer cell lines, including 2 lung adenocarcinoma cell lines (H23 and H838) $(11,15)$ and uterine cervical cancer cells (HeLa) were used in the study. Cells were maintained at $37^{\circ} \mathrm{C}$ as a monolayer in RPMI-1640 supplemented with $10 \%$ fetal calf serum, $100 \mu \mathrm{g} / \mathrm{ml}$ of streptomycin and $100 \mathrm{IU} / \mathrm{ml}$ of penicillin. To generate cells that remained at the $\mathrm{G} 1 / \mathrm{G} 0$ phase of the cell cycle, $\mathrm{H} 23$ cells were grown to $70 \%$ confluence from the initial seeding and changed to medium containing $0.5 \%$ of serum for $48 \mathrm{~h}$. For HeLa cells to be at the different phases of cell cycle, the cells were respectively treated by serum starvation, double thymidine block or thymidine-nocodazole. To enrich cells at the $\mathrm{S}$-phase, cell cycle progression of HeLa cells were blocked with $2 \mathrm{mM}$ thymidine for $18 \mathrm{~h}$, before a release for $9 \mathrm{~h}$. The cells were then treated with a second thymidine block for $15 \mathrm{~h}$ and a release for $6 \mathrm{~h}$. The enriched G2/M cells were obtained by thymidine-nocodazole treatment. The cell cycle was blocked with $2 \mathrm{mM}$ thymidine for $24 \mathrm{~h}$, a release for $3 \mathrm{~h}$, and incubated with $100 \mathrm{ng} / \mathrm{ml}$ nocodazole for $12 \mathrm{~h}$. The cells were collected and stained with propidium iodide before analysis by flow cytometry (Beckman Coulter Cytomics ${ }^{\mathrm{TM}}$ FC500).
Fractionation of cellular components. Fractionation of subcellular components was performed according to the instruction manuals of Calbiochem (http://www.merckbiosciences.co.uk) with minor modifications. The cells were detached from culture plates by treatment with dissociation buffer (Sigma, St. Louis, MO) at $37^{\circ} \mathrm{C}$ for 2-5 min. After washing with PBS, the cells were re-suspended in $\mathrm{H}$ buffer $(10 \mathrm{mM}$ Tris- $\mathrm{HCl}, \mathrm{pH}$ 7.4, $10 \mathrm{mM} \mathrm{KCl}, 1.5 \mathrm{mM} \mathrm{MgCl}_{2}, 1 \mathrm{mM}$ Na-EDTA, $1 \mathrm{mM}$ Na-EGTA, $1 \mathrm{mM}$ dithiothreitol, $250 \mathrm{mM}$ sucrose, $0.1 \mathrm{mM}$ PMSF, $10 \mu \mathrm{g} / \mathrm{ml}$ leupeptin, $10 \mu \mathrm{g} / \mathrm{ml}$ aprotinin and $10 \mu \mathrm{g} / \mathrm{ml}$ trypsin inhibitor) at $4^{\circ} \mathrm{C}$ for $15 \mathrm{~min}$. After 80 strokes with a $B$ pestle in a Douncer homogenizer, the unbroken cells were removed by centrifugation at $30 \mathrm{x} g$ for $5 \mathrm{~min}$. The nuclei were collected by spinning the solution at $80 \mathrm{x}$ g for $10 \mathrm{~min}$, and mitochondria were collected from the supernatant by centrifugation at $6,000 \mathrm{x} \mathrm{g}$ for $20 \mathrm{~min}$. Following centrifugation at $20,000 \mathrm{x} g$ for $20 \mathrm{~min}$ to remove the insoluble debris, the final supernatant was used as a post-mitochondrial fraction (15). For isolating MAM and microsomes, the homogenates were centrifuged twice at $600 \mathrm{x}$ g to completely remove nuclei. The supernatant was centrifuged at $10,500 \mathrm{x} g$ to separate the crude microsomal (microsomes and cytosol) from the crude mitochondrial (MAM and mitochondria) fractions. The crude microsomal fractions (supernatant) were subjected to an ultracentrifugation at $100,000 \mathrm{x} \mathrm{g}$ for $60 \mathrm{~min}$ at $4^{\circ} \mathrm{C}$ to pellet the microsomes, while the supernatant was used as cytosol. The crude mitochondrial fractions (pellet) were resuspended in $300 \mu \mathrm{l}$ of ice-cold mannitol buffer A $(0.25 \mathrm{M}$ mannitol, 5 mM HEPES, 0.5 mM EGTA, pH 7.2) and layered on top of $10 \mathrm{ml}$ of a $30 \%$ Percoll suspension in mannitol buffer B (0.25 M mannitol, 25 mM HEPES, 1 mM EGTA, pH 7.2). Mitochondria and MAM fractions were separated during the formation of self-generating Percoll gradient by ultracentrifugation at 95,000 $\mathrm{x} \mathrm{g}$ for $65 \mathrm{~min}$ at $4^{\circ} \mathrm{C}$. Both isolated fractions were diluted 5 times in sucrose homogenization medium and subjected separately to a centrifugation at $6,300 \mathrm{x} \mathrm{g}$ for $10 \mathrm{~min}$ at $4^{\circ} \mathrm{C}$. The pellet was used as the purified mitochondria, while the supernatant was further separated by centrifugation at $100,000 \times \mathrm{g}$ for $30 \mathrm{~min}$ at $4^{\circ} \mathrm{C}$, and the pellet was used as the purified MAM fraction. The ultracentrifugation was performed using the Beckman SW41 rotor, and all of the fractions were resuspended in sucrose homogenization medium before immunoblotting analysis.

Immunoblotting and immunofluorescent staining. The procedure for immunoblotting has been previously described (10). Briefly, proteins were separated on a $10 \%$ polyacrylamide gel with $4.5 \%$ stacking. After electrophoresis, proteins were transferred to a nitrocellulose membrane. The membrane was then probed with specific antibodies. The signal was amplified by biotin-labeled goat anti-mouse IgG, and peroxidase-conjugated streptavidin. The protein was visualized by exposing the membrane to an X-Omat film (Eastman Kodak, Rochester, NY) with enhanced chemiluminescent reagent (NEN, Boston, MA). Following characterization with immunoblotting, the same antibodies were used for immunofluorescence confocal microscopy. For the staining, cellular uptake of MitoTracker ${ }^{\circledR}$ green FM (Molecular Probes, Inc., Eugene, OR) was used to label mitochondria. The cells were then fixed with $4 \%$ formaldehyde at room temperature for $15 \mathrm{~min}$. After washing 3 times 
A

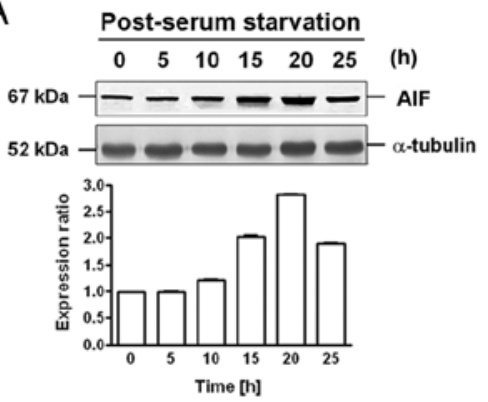

C

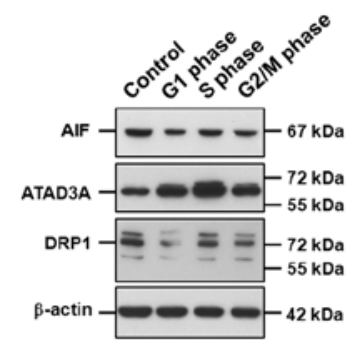

B

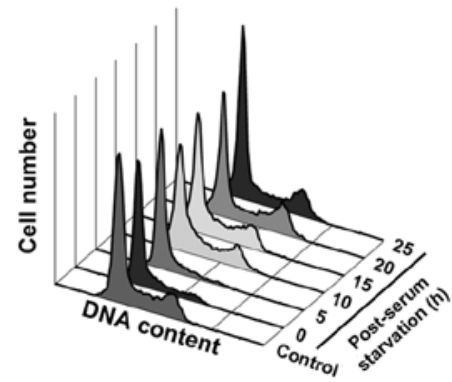

D

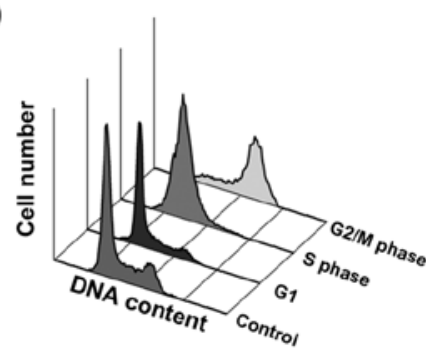

Figure 1. Expression level of AIF increases during the proliferation phase of the cell cycle. (A) Expression levels of AIF as measured by immunoblotting. Whole cell lysate $\left(5 \times 10^{6}\right.$ cells/lane) was prepared from quiescent $\mathrm{H} 23$ cells (Time 0$)$ and serum-stimulated cells at 5-h intervals for $25 \mathrm{~h}$. The expression ratio of AIF, which was measured by density scanning of the exposed films, is shown in the lower panel. (B) Flow cytometry was used to analyze cell cycle progression of $\mathrm{H} 23$ cells, which were synchronized by serum starvation for $48 \mathrm{~h}$ and then released by addition of fresh serum. The harvest intervals were $5 \mathrm{~h}$. (C) Expression levels of AIF at the different phases of the cell cycle in HeLa cells. G1-phase cells were collected following serum starvation for $48 \mathrm{~h}$, S-phase cells were collected $6 \mathrm{~h}$ following release from double thymidine block, and G2/M-phase cells were collected from thymidine-nocodazole treatment. (D) Flow cytometry was used to analyze cell cycle progression of HeLa cells, which were harvested from the control, serum starvation, double thymidine block and release, and thymidine-nocodazole co-treatment.

with PBS, cells were incubated with the primary antibodies for $90 \mathrm{~min}$, and washed 3 times with PBS. The secondary antibodies used were rhodamine (TRITC)-conjugated rabbit anti-mouse IgG (Jackson ImmunoResearch Laboratories, Inc., West Grove, PA). Nuclei were stained with 4',6-diamidino2-phenylindole (DAPI). The slide was examined and the images were captured with a laser scanning confocal microscope (LSM510, Zeiss, Chicago, IL). The images were processed by a Zeiss LSM Image Browser (LSM5 Image Software, Zeiss, Chicago, IL) and the Photoshp 7.0 software (Adobe Systems Incorporated).

Construction of AIF-expressing plasmids. The forward and reverse primer sequences for the cloning expression vector containing the full-length AIF (AF100928, coding sequence, nts 179-2020) were the following: 5'-TTGGAATTCATTA TGTTCCGGTGTGGAGGCCTG-3' (nts 43-63, EcoRI site is underlined and the initiation codon is in bold); and 5'-TAC CGTCGACTGTGAAATTCAGTCTTCATGAATGT-3' (nts 1869-1884, SalI site is in italic and underlined and the stop codon is in bold). The amplified DNA fragments were digested with the respective restriction enzymes and then inserted into the vector pcDNA3.1.

Electron microscopy. Electron microscopy was carried out using a routine protocol (15). Briefly, cells were fixed with $2.5 \%$ glutaraldehyde (EM grade, Sigma, St. Louis, MO, USA) in $100 \mathrm{mM}$ phosphate buffer (PB, $\mathrm{pH} 7.2)$, incubated at $4^{\circ} \mathrm{C}$ overnight. The cells were washed with $\mathrm{PB} 3$ times before post-fixation with $1 \%$ osmium tetroxide in PB for $2 \mathrm{~h}$. After removal of the fixative with distilled water, the cells were suspended in $2 \%$ molten agar. The agar blocks were trimmed and dehydrated in a serial dilution of ethanol for $15 \mathrm{~min}$ each. The blocks were further dehydrated with $100 \%$ ethanol for 15 min 3 times, and infiltrated with $100 \%$ ethanol/LR White ${ }^{\mathrm{TM}}$ (1:1) mixture overnight. The blocks were changed to the pure LR White (Agar Scientific Ltd., Essex, UK), continued infiltration at $4^{\circ} \mathrm{C}$ for $24 \mathrm{~h}$, before transfer to a capsule filled with $\mathrm{LR}$ White, and were polymerized and solidified at $60^{\circ} \mathrm{C}$ for $48 \mathrm{~h}$. The resin blocks were trimmed and cut with an ultramicrotome (Leica Ultracut R, Leica Mikrosysteme GmbH, Vienna, Austria). The thin sections were transferred to 200 mesh copper grids, and stained with $2 \%$ uranyl acetate for $30 \mathrm{~min}$, and $2.66 \%$ lead citrate $(\mathrm{pH} 12.0)$ for $10 \mathrm{~min}$, before observation with an electron microscope (JEM1400, Jeol USA, Inc., Peabody, MA) at 100-120 kV. For gene silencing experiments, cells were harvested $48 \mathrm{~h}$ following siRNA treatment.

Post-embedded immune-gold procedure for electron microscopy. Following fixation with $4 \%$ paraformaldehyde and $0.1 \%$ glutaraldehyde in PBS buffer at $4^{\circ} \mathrm{C}$ for $18 \mathrm{~h}$, the cells were washed with PBS 3 times. The cells were then permeabilized with $0.2 \%$ Triton X-100 in PBS for $20 \mathrm{~min}$. After being washed with PBS for 3 times, the cells were embedded in $2 \%$ agarose and the agarose block was cut into several pieces prior to dehydration. After dehydration in a series of ethanol with escalated concentration, samples were embedded in LR White resin. Thin sections were cut with an ultramicrotome and transferred to nickel grids. The non-specific binding sites on the thin sections were blocked with $50 \mathrm{mM}$ glycine and $1 \%$ BSA in PBS for $15 \mathrm{~min}$. The sections were incubated with the primary antibody (1:50) in incubation buffer $(0.1 \%$ 
A

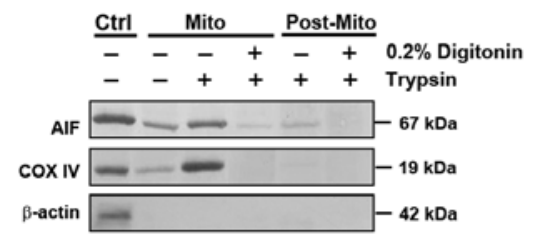

B

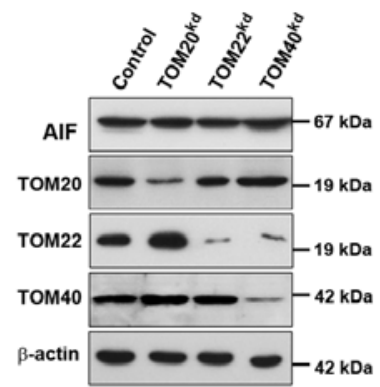

C

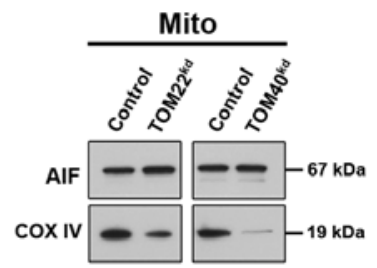

Figure 2. AIF is protected by digitonin-sensitive molecules and is not imported into mitochondria through TOM complexes. (A) HeLa cells were synchronized at the S-phase, and protein was collected from mitochondrial (mito) and post-mitochondrial (post-mito) fractions. AIF from both of these 2 fractions was resistant to trypsin (in $0 \%$ digitonin), while AIF became sensitive to trypsin after $0.2 \%$ of digitonin pre-treatment. These data suggest that in the S-phase AIF is protected by trypsin-resistant molecules. (B) Immunoblot analysis of total cell lysates which were from the wild-type, TOM $20^{\mathrm{kd}}$, TOM $22^{\mathrm{kd}}$, and TOM $40^{\mathrm{kd}}$ H838 cells. (C) Immunoblot analysis of mitochondrial fractions which were from the wild-type, TOM $22^{\mathrm{kd}}$ and TOM40 ${ }^{\mathrm{kd}} \mathrm{H} 838$ cells. Each experiment was repeated at least 3 times.

A

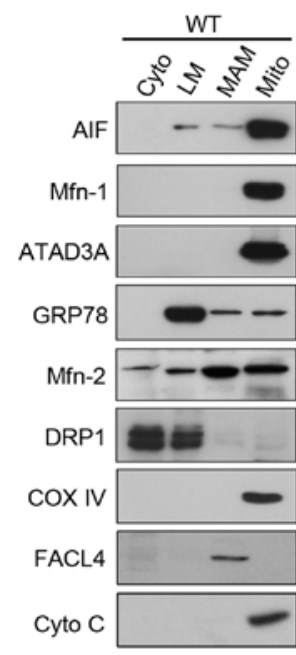

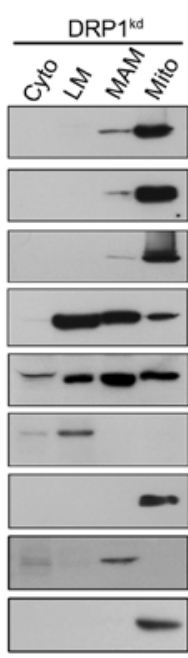

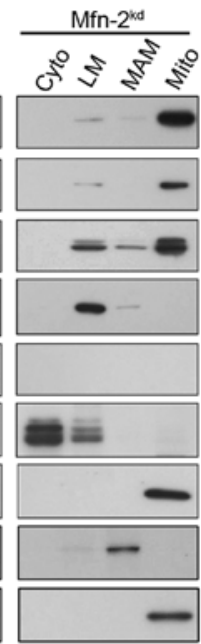

B

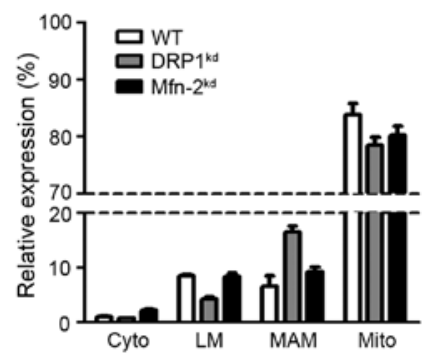

C

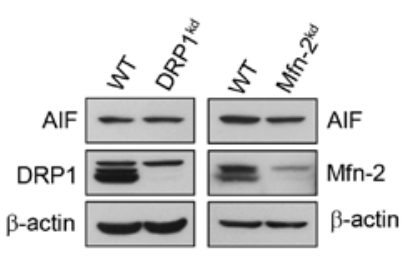

Figure 3. Immunoblot analysis of mitochondria-associated proteins in subcellular fractions. (A) Immunoblot analysis of membrane fractions isolated by sucrose gradient ultracentrifugation in the wild-type (WT, the left panel), the DRP1 $1^{\mathrm{kd}}$ (the central panel) or Mfn- $2^{\mathrm{kd}}$ (the right panel) human lung cancer H838 cells. WT, wild-type; Cyto, cytosolic fraction; LM, light membrane; MAM, mitochondria-associated membrane; Mito, mitochondrial fraction. Fatty-acid CoA ligase 4 (FACL4) is a MAM marker; cytochrome oxidase IV (COX IV) is a mitochondrial inner membrane marker; cytochrome c (Cyt c) is a mitochondrial intermembranous space marker. (B) The subcellular distribution of AIF in DRP $1^{\mathrm{kd}}$ and Mfn- $2^{\mathrm{kd}}$ cells. Results of immunoblotting were normalized to the total amount of AIF. Levels of AIF increased in the MAM fractions, but reduced in the mitochondrial fractions in both DRP1 ${ }^{\mathrm{kd}}$ and Mfn- ${ }^{\mathrm{kd}}$ cells. (C) Immunoblot analysis of total proteins in WT, DRP1 ${ }^{\mathrm{kd}}$ or Mfn- $2^{\mathrm{kd}}$ human lung cancer $\mathrm{H} 838$ cells. Each experiment was repeated at least 3 times.

BSA and $15 \mathrm{mM} \mathrm{NaN}_{3}$ in PBS) for $2 \mathrm{~h}$ at room temperature. The antibodies were then removed by washing the grids with incubation buffer for 6 times and the grids were further incubated with $15 \mathrm{~nm}$ gold-conjugated secondary antibody (1:25) (Aurion, Wageningen, The Netherlands). Following removal of the non-binding immune-gold by repeated washing of the grids with incubation buffer, the sections were post-fixed with 2\% glutaraldehyde in PBS for 15 min and stained with uranyl acetate and lead citrate before observation with an electron microscope.

\section{Results}

Newly expressed AIF, the levels of which increase during the $S$ and $G 2$ phases of the cell cycle, is resistant to trypsin, and is detected not only in mitochondria. The biogenesis of mitochondria occurs in the S phase (17). We therefore anticipated that biosynthesis of AIF was cell cycle-dependent. Serum-starved quiescent $\mathrm{H} 23$ cells, when replenished with fresh serum, synchronously entered cell cycle progression (18). Levels of AIF increased about $10 \mathrm{~h}$ after serum stimulation, and reached a peak at $20 \mathrm{~h}$ (Fig. 1A). The results were verified by flow cytometry analysis, in which AIF levels increased in the $\mathrm{S}$ and $\mathrm{G} 2$ phases of the cell cycle (Fig. 1B-D).

Several mitochondria-associated proteins, e.g., DRP1 and ATAD3A, also increased during these phases (Fig. 1C) $(18,19)$. Interestingly, in addition to the mitochondrial fractions, AIF was also detected in post-mitochondrial fractions (Fig. 2A), and AIF from these 2 fractions was resistant to trypsin in synchronized S and G2 cells. However, AIF became sensitive to trypsin when the post-mitochondrial fractions were pretreated with digitonin (Fig. 2A), suggesting that AIF in the 
A
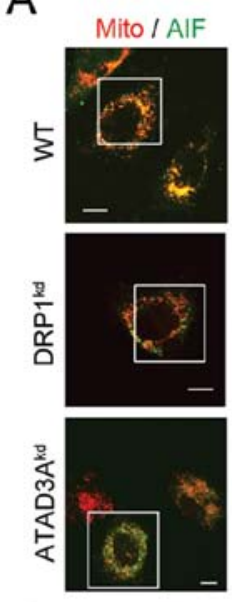

C

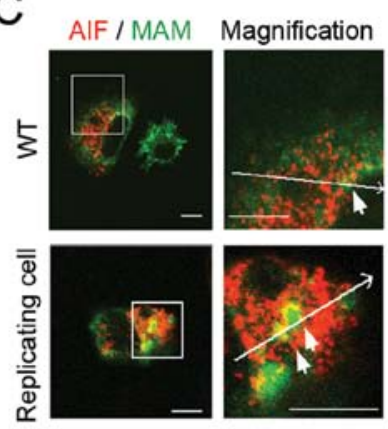

Magnification
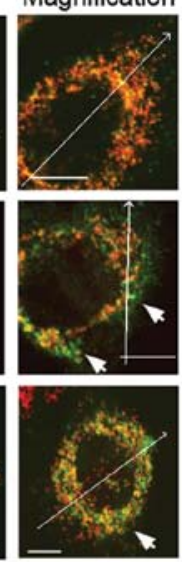

Magnification

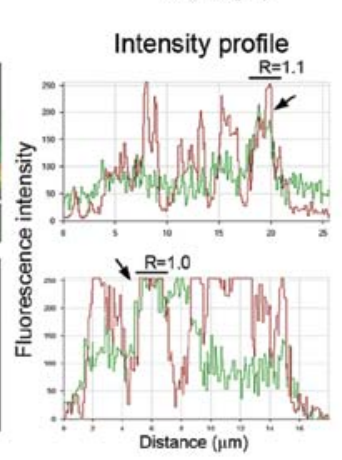

B

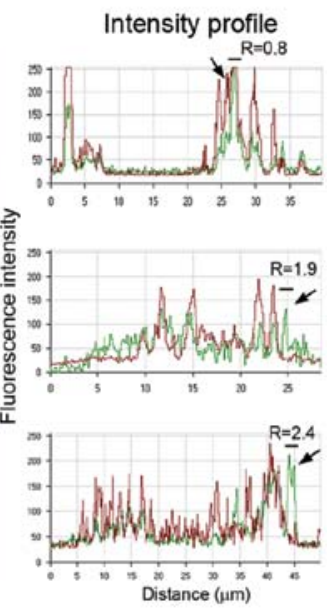

D
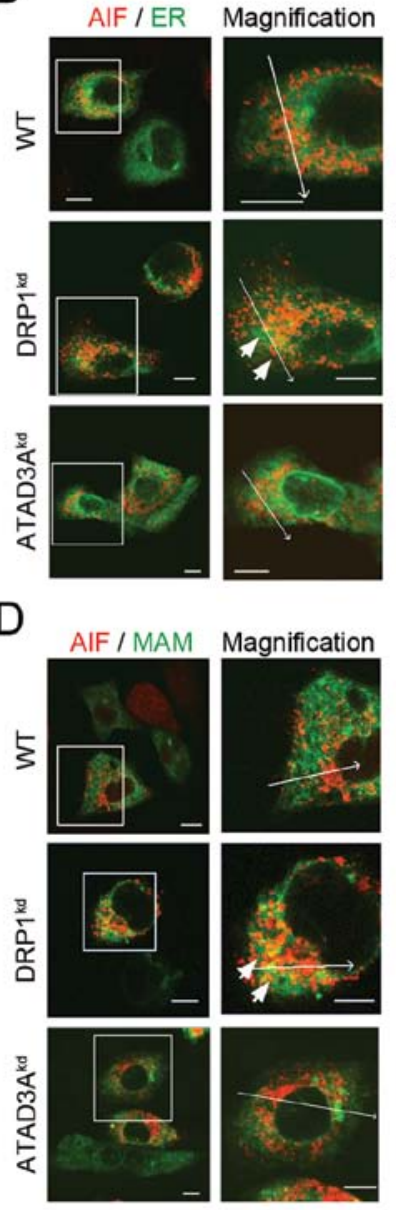

Intensity profile
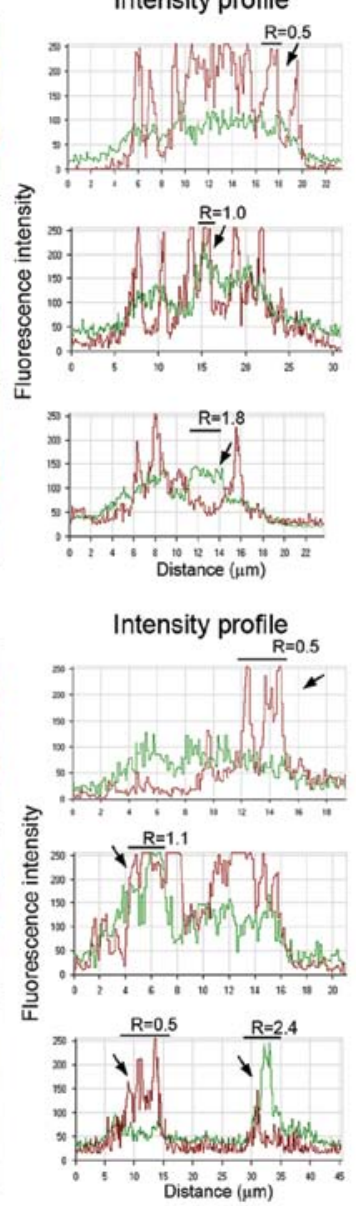

Figure 4. The effect of mitochondrial import-related protein expression on the subcellular distribution of AIF as determined by confocal immunofluorescence microscopy. (A) Compared to the wild-type H838 cells, knockdown of DRP1 (DRP1 ${ }^{\mathrm{kd}}$, central row) or ATAD3A expression increased the cytoplasmic level of recombinant human AIF (rhAIF) (arrows, green fluorescence). Mitochondria were stained with DsRed. The images were processed by a Zeiss LSM Image Browser (LSM5 Image Software, Zeiss, Chicago, IL). R, ratio of green/red fluorescence; if R=1, the intensity of the green fluorescence equals that of the red fluorescence, and the green fluorescence overlaps with the red fluorescence. Overlapped fluorescence appears in yellow. If $\mathrm{R}>1$, the intensity of the green fluorescence is stronger than that of the red fluorescence, resulting in a green signal. If $R<1$, the green fluorescence is weaker than the red fluorescence, and the fluorescence signal appears red. (B) Silencing of DRP1, but not that of ATAD3A, increases co-localization of rhAIF (arrows, yellow fluorescence) and the endoplasmic reticulum (ER-GFP), suggesting that rhAIF was present in the ER. (C) In the replicating H838 cells, some of the rhAIF is localized in the bulging MAM (arrows, yellow fluorescence), as visualized by a transiently expressed huPSS1-GFP, indicating that AIF is abundantly present in the MAM of the replicating H838 cells (H838/Repl). (D) In DRP $1^{\mathrm{kd}}$ cells, transiently expressed huPSS1-GFP co-localized with the rhAIF in the bulging MAM, suggesting that ectopically expressed AIF accumulates in the enlarged MAM when the formation mechanism of the transport vesicles was inhibited. Silencing of ATAD3A reduces both the size of the ER (green fluorescence) and of the mitochondria. These results are representative of 3 independent experiments.

$\mathrm{S}$ and G2 phases could be protected by digitonin-sensitive molecules, e.g., phospholipids or inside vesicles.

In general, the mitochondrial proteins are believed to be imported into mitochondria via the TOM40 complex. Deficiency of TOM40 components will reduce protein levels of the mitochondria. However, we did not detect the evident reduction of AIF in the total cell lysate or mitochondrial fractions when expression of TOM40 channel or TOM22 receptor was silenced (Fig. 2B and C), suggesting that AIF might not be imported through the TOM40 translocator of the mitochondria. These results in part support our hypothesis that AIF might not be imported to mitochondria via the routine TOM passage, and in part suggest that there might be an alternative protein transport pathway to mitochondria.

Subcellular localization of AIF as determined by immunoblotting. Because results of trypsin digestion suggested that
AIF could be present within the phospholipids, we then used sucrose gradient ultracentrifugation and immunoblotting to determine the membrane fractions of lung cancer cells in which AIF was present. As shown in Fig. 3A, AIF and the 78-kDa glucose-regulated protein (GRP78) were detected in the ER the light membrane (LM) fraction, MAM and the mitochondrial fractions of the wild-type H838 cells, but not in the cytosol. Knockdown of the 80-kDa DRP1 (DRP1 ${ }^{\mathrm{kd}}$ ) protein expression, decreased levels of AIF, ATAD3A, GRP78, Mfn-1 and -2 in the fractions of mitochondria, the target organelle of these proteins (Fig. 3A and B, the middle panel). However, levels of these proteins increased in the fractions of MAM, suggesting that DRP1 expression affected the transport of these proteins from the ER to the mitochondria, and that the MAM could be a docking area for the shipment of these cargo proteins to the mitochondria. These results together with our previous data indicate that DRP1 could play a role in regulating 
the budding of transport vesicles from MAM (10). Knockdown of Mfn-2 (Mfn- $2^{\mathrm{kd}}$ ) expression, on the other hand, decreased levels of AIF, Mfn-1 and GRP78 in the mitochondrial fractions (Fig. 3A and B, right panel), indicating that Mfn-2 was essential for the fusion of transport vesicles into mitochondria. These results clearly showed that knockdown of DRP1 or Mfn-2 could interfere with AIF distribution in the ER, the MAM and the mitochondria. Silencing of import-related protein expression would leave the cargo protein AIF build up in the prior membrane fractions, i.e., the ER, the MAM and the transport vesicles. Temporally knockdown of DRP1 or Mfn-2 did not markedly affect total AIF protein levels (Fig. 3C), however, caused re-distribution of AIF in the different organelles, in particular accumulation in the previous organelles (10).

Subcellular localization of AIF as determined by confocal microscopy. To further verify our hypothesis, we used confocal immunofluorescence microscopy to examine the distribution pattern of full-length recombinant human AIF in cells. As anticipated, rhAIF was mainly localized in the mitochondria (mitochondria were visualized by live dye of mitochondria, DsRed-Mito; Fig. 4A, upper panel). In DRP $1^{\mathrm{kd}}$ cells, some of rhAIF did not co-localize with mitochondria (Fig. 4A, central panel), but with the ER and MAM (ER was visualized by the ER-retention signal KDEL-conjugated GFP; Fig. 4B, central panel). In ATAD3A ${ }^{\mathrm{kd}}$ cells, the rhAIF was mainly present in the cytoplasmic vesicles (Fig. 4A and B, lower panel).

To further differentiate whether the co-localization was in the ER or MAM, we labeled the MAM with human phosphotidylserine synthase 1 (huPSS1)-conjugated GFP. In the resting cells, no evident co-localization was detected between rhAIF and the MAM (Fig. 4C, upper panel). In replicating cells, few areas of co-localization between rhAIF and the MAM were detected (Fig. 4C, lower panel). In DRP $1^{\mathrm{kd}} \mathrm{H} 838$ cells, co-localization between the MAM and rhAIF became evident (Fig. 4D, central panel), suggesting that inhibition of budding off of transport vesicles from the MAM accumulated cargo proteins, such as AIF, in the docking mitochondria-associated membrane. In ATAD3A $\mathrm{A}^{\mathrm{kd}}$ cells, owing to the lack of moving ability, the transport vesicles which had been budded off from the MAM would be dispersed around the MAM.

Subcellular localization of AIF as determined by immunegold electron microscopy. To gain further insight, electron microscopy was employed to determine the ultrasubcellular localization of AIF. AIF-specific immune-gold particles were identified in the cytoplasm, the ER, the transport vesicles, and the mitochondria (Fig. 5A and B). Silencing of DRP1 (DRP1 ${ }^{\mathrm{kd}}$ ) expression increased the average length of mitochondria and the formation of the bulging mitochondria-associated membranes (or the ER). Knockdown of Mfn-2 (Mfn- $2^{\mathrm{kd}}$ ) expression, on the other hand, increased the amount of transport vesicles. Ultrastructurally, AIF accumulated in the dilated ER/MAM (Fig. 5C and D). Interestingly, in ATAD3A ${ }^{\mathrm{kd}}$ cells, the AIF signal was more evident in transport vesicles (Fig. 5E and F). In Mfn- $2^{\mathrm{kd}}$ cells, however, the AIF signals were mainly present in transport vesicles around the mitochondria (Fig. 5G and $\mathrm{H}$ ), suggesting that other than mitochondrial fusion, Mfn-2 might play a role in maintaining the ER integrity and probably in fusion of transport vesicles into the mitochondria. These

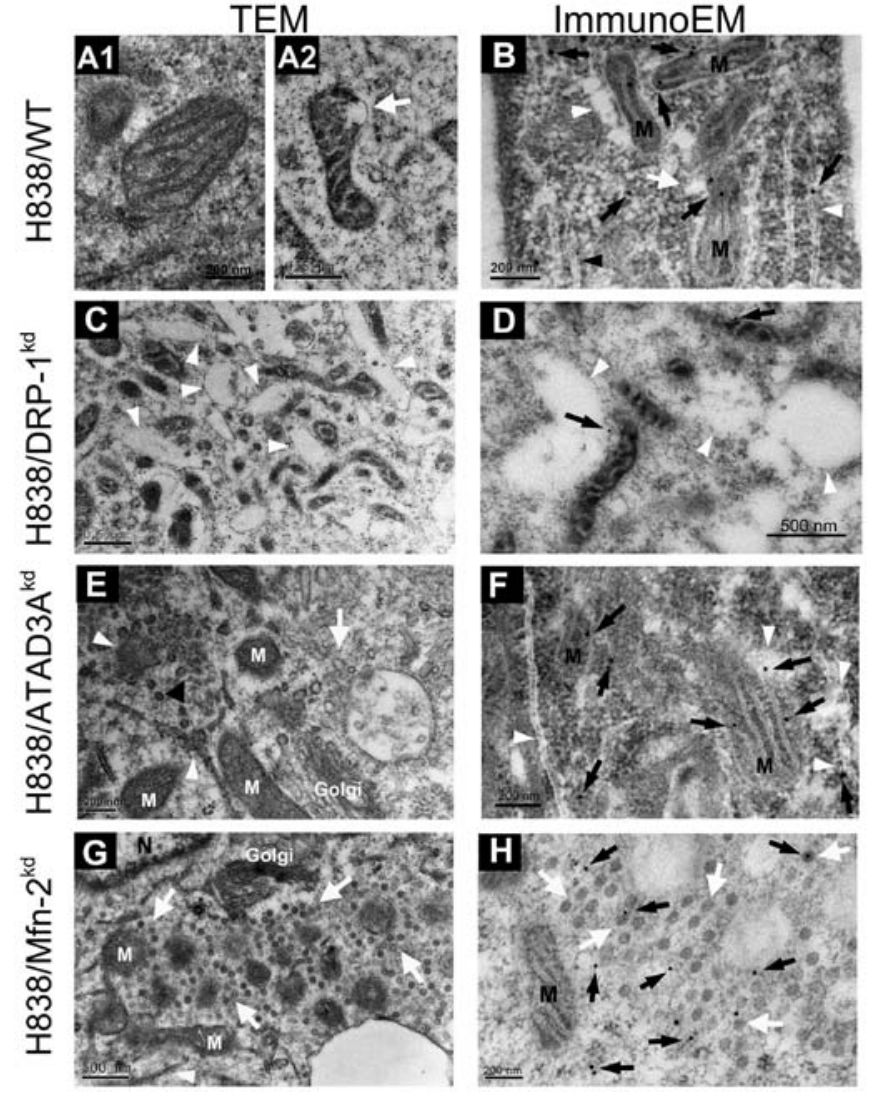

Figure 5. The effect of mitochondrial protein import-related protein expression on the subcellular distribution of AIF as determined by electron microscopy. (A) Electron microscopy analysis of the wild-type H838 cells showed (A1) the fusion of transport vesicles (white arrows) and (A2) into mitochondria, which were about 50-70 $\mathrm{nm}$ in diameter. (B) Using electron microscopy, the AIF-specific immune-gold particles were identified in the transport vesicles (black arrows), the transport vesicles (white arrows) that were fusing with the mitochondrion. (C) Silencing of DRP1 (DRP1 ${ }^{\mathrm{kd}}$ ) expression increased the number of bulging MAM, (white arrowheads) and protein aggregates at the margin of the bulging MAM. (D) Using immune-gold, AIF (black arrows) was detected in protein aggregates of the dilated ER/MAM (white arrowheads) in DRP $1^{\mathrm{kd}}$ cells. (E) Silencing of ATAD3A (ATAD3A ${ }^{\mathrm{kd}}$ ) expression increased the number of fragmented mitochondria (black arrowheads) and transport vesicles (white arrows) around the dilated ER/MAM (black arrow). (F) In ATAD3 $\mathrm{A}^{\mathrm{kd}}$ cells, the AIF signal was mainly present in the transport vesicles (black arrow) in the cytoplasm or around the ER/MAM (white arrowheads). (G) In Mfn- $2^{\mathrm{kd}} \mathrm{H} 838$ cells, the presence of transport vesicles (white arrows) was more evident. M, mitochondria; N, nucleus; (H) The AIF signals were detected in the prospective transport vesicles in Mfn- $2^{\mathrm{kd}} \mathrm{H} 838$ cells (black arrows), confirming our hypothesis that DRP1, ATAD3A, and Mfn-2 were essential for the shaping of the ER and the mitochondrial, in particular in the shipment of transport vesicles containing AIF from the ER/MAM to the mitochondria. These results are representative of 3 independent experiments, in which about 10-50 cells were analyzed.

results confirmed our hypothesis that DRP1, ATAD3A, and Mfn-2 were essential to maintain shaping of the ER, MAM and the mitochondria, in particular in the shipment of transport vesicles, which were budding off from the ER/MAM, and were imported into the mitochondria.

\section{Discussion}

The results presented above support our hypothesis that AIF is imported from the ER to the mitochondria, probably via MAM and transport vesicles. This protein transport pathway 


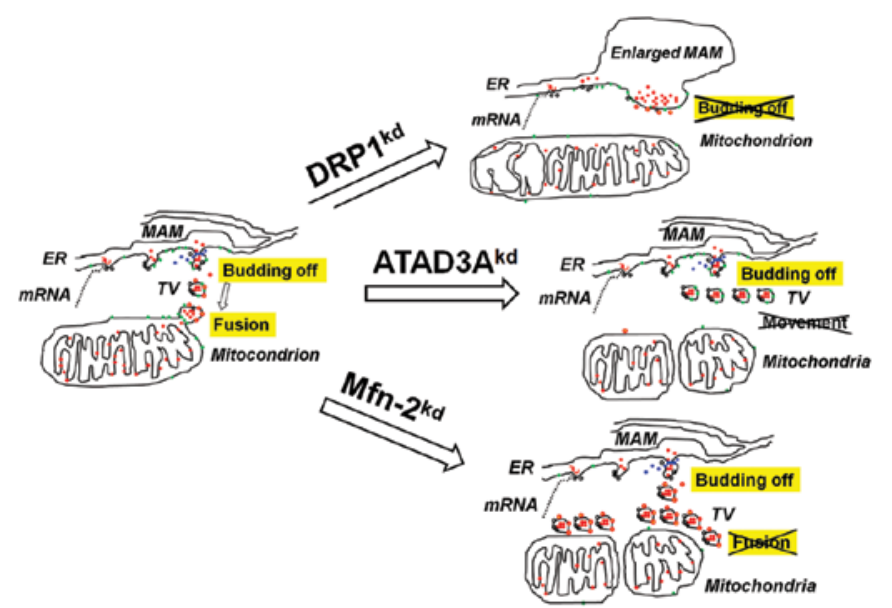

AIF; OATAD3A; O eEF2; DRP-1; Mfn-2

Figure 6. A simplified diagram of the import route of AIF to the mitochondria. The import of AIF is from the ER, the MAM, transport vesicles to the mitochondria (the left panel). In DRP1 ${ }^{\mathrm{kd}}$ cells, because the budding off mechanism of the transport vesicles is inhibited, the accumulated cargo proteins and, possibly, the phospholipids, which are detained in the ER as well as in the MAM, will cause the bulging of the MAM (upper right panel). In ATAD3A ${ }^{\mathrm{kd}}$ cells, although the transport vesicles are formed around the dilated ER/MAM, the lack of ATAD3A (ATPase for providing movement energy) will idle movement of transport vesicles toward the mitochondria Deficiency in the supplement of newly synthesized proteins and phospholipids will then induce mitochondrial fragmentation and increase the number of fragmented mitochondria (the middle right panel). Likewise, because the transport vesicles cannot fuse into the mitochondria, the presence of transport vesicles around mitochondria is more evident in $\mathrm{Mfn}-2^{\mathrm{kd}}$ cells. The markedly increased transport vesicles around mitochondria may be induced by a yet to be determined intracellular feedback mechanism that is essential for maintaining the morphology and function of the ER, MAM and mitochondria.

requires at least 3 proteins: DRP1, ATAD3A and Mfn-2. DRP1 is important for the formation of transport vesicles from MAM, while ATAD3A is essential for delivering transport vesicles to the mitochondria, and Mfn-2 is critical for the fusion of transport vesicles into the mitochondrial outer membrane. A simplified diagram of this mechanism is shown in Fig. 6. In this case, cells can import AIF as well as other proteins and phospholipids from MAM to the mitochondria.

Our previous study clearly demonstrated such a possibility (10). By using antibodies specific to ATAD3A, we precipitated AIF in detergent-free post-mitochondrial fractions, but not in cytosolic fractions. Moreover, antibodies specific to ATAD3A precipitated the eukaryotic elongation factor-2 (eEF-2), Mfn-2, DRP1 and OPA1 as well, indicating that these molecules interact with one another in the post-mitochondrial fractions (10). However, antibodies specific to AIF only precipitated AIF in a cell lysate containing nonionic detergent NP-40, but not in detergent-free post-mitochondrial fractions (data not shown), suggesting that AIF was prevented from binding to antibodies by NP-40 sensitive molecules, such as phospholipids.

Interestingly, mitochondria do not synthesize phospholipids de novo. Essential phospholipid, e.g., phosphatidylserine (PS), is synthesized in the ER and then transported to the mitochondria before converting to phosphatidylethanolamine (PE). A study by Shiao et al showed that phospholipid exchange between the ER and the mitochondria was protein-dependent (19). They suggested that phospholipid exchange might possibly occur via MAM, a specialized area of the ER which is in close contact with the mitochondria, and had been implicated in regulating phospholipid biosynthesis and transport, calcium transmission, energy metabolism and cell survival (20-22). By studying biosynthesis of phosphatidylcholine (PC), de Kroon et al showed that phospholipid import required both ATP and GTP (23). In addition, DRP1 was not only detected on the ER and the mitochondria, but also on a number of small cytoplasmic vesicles (14). Expression of a dominant negative $\mathrm{DRP} 1^{\mathrm{K} 38 \mathrm{~A}}$ gene markedly reduced the number of cytoplasmic vesicles and induced bulging of the mitochondria-associated membranes, supporting our data that proteins and phospholipids could form cargo vesicles in the MAM (13-15,24). It is worth noting that biogenesis of mitochondria occurs at the S-phase. Expression of many mitochondrial proteins, e.g., cytochrome c oxidases, is also cell cycle-dependent and regulated by nuclear respiratory factor (Nrf) $(25,26)$. These proteins were continuously synthesized in the cytoplasm; otherwise, they would swarm the mitochondrial periphery and aggregate at the TOM/TIM complexes.

Lately, Mfn- 2 was identified as a linker protein on the MAM and the mitochondria, which tethered the 2 organelles together (12). In addition to mitochondrial fusion, Mfn-2 was imperative for regulation of $\mathrm{Ca}^{2+}$ flow between the ER and mitochondria $(10,27)$. Nevertheless, besides a cleavage site for mitochondrial presequence and 2 peroxisomal targeting signals, no evident mitochondrial targeting sequence was detected in Mfn-2 protein. Because the peroxisome is derived from the ER, and formation of the peroxisome occurs via MAM, which requires DRP1 and AAA-ATPases (Pex1 and Pex6) $(28,29)$, findings by de Brito and Scorrano $(12,13)$ indicate that targeting of Mfn- 2 to the ER and mitochondria is either mediated by a yet to be determined mechanism or import of some mitochondrial proteins is by an alternative route $(15,30)$, which conceivably is via MAM and the transport vesicles.

By studying hepatitis c virus core protein (HCVCP), Schwer et al showed that HCVCP was frequently detected in the ER, the light membrane, MAM and mitochondria. By investigating intracellular trafficking of human cytomegalovirus (hCMV) UL37 proteins, Bozidis et al found that the full-length UL37 protein (gpUL37) and the derivatives were all identified in the MAM and sometimes in mitochondria (31). Studies by Burikhanov et al and Sun et al showed that GRP78, a chaperone protein of the ER, was also detected in the mitochondria, nucleus and plasma membrane $(32,33)$. These results considered together with our current data clearly suggest that sorting of some mitochondrial proteins may take an alternative route, from the ER and MAM. In this way, phospholipids and proteins could be simultaneously transported from the ER to mitochondria without causing imbalance between the quantity of phospholipids and proteins in target organelles. Our hypothesis provides a reasonable explanation for why silencing of any of the 3 genes, DRP1, ATAD3A or Mfn-2, concomitantly alters morphology of the ER, MAM and mitochondria, and in severe cases it induces autophagy.

In conclusion, we identified the passage way for AIF transport from the ER to mitochondria. This process requires at least 3 proteins, DRP1, ATAD3A and Mfn-2, each of which has a different role: DRP1 is required for forming transport vesicles from the MAM, ATAD3A is involved in shipping 
of the transport vesicles to the mitochondria, and Mfn- 2 is involved in the fusion of transport vesicles into the mitochondria (Fig. 6). It should be noted, however, that other proteins could be implicated in this process (30). In an ongoing study, we are investigating how many mitochondrial proteins are imported via this route.

\section{Acknowledgements}

We thank DrF.Peruzzi, Department of Neuroscience and Center for Neurovirology, Temple University School of Medicine, USA, for DsRed-Mito; Dr D.J. Snyders, Department of Biomedical Sciences, University of Antwerp, Belgium, for DsRed-ER; Dr Jiuping Ding and Dr Tao Xu, Key Laboratory of Molecular Biophysics, Huazhong University of Science and Technology, People Republic of China, for pEGFP-ER and Dr Anamaris M. Colberg-Poley, Center for Cancer and Immunology Research, Children's Research Institute, Children's National Medical Center, USA, for mEGFP-huPSS-1. This study was supported, in part, by the Comprehensive Academic Promotion Projects (NCHU 995002, Ministry of Education) and, in part, by the Cancer Excellent Research System (DOH100-TD-111-005, Taiwan).

\section{References}

1. Ahmed AU and Fisher PR: Import of nuclear-encoded mitochondrial proteins: a cotranslational perspective. Int Rev Cell Mol Biol 273: 49-68, 2009.

2. Chacinska A, Koehler CM, Milenkovic D, Lithgow T and Pfanner N: Importing mitochondrial proteins: machineries and mechanisms. Cell 138: 628-644, 2009.

3. Murakami K, Amaya Y, Takiguchi M, Ebina Y and Mori M: Reconstitution of mitochondrial protein transport with purified ornithine carbamoyltransferase precursor expressed in Escherichia coli. J Biol Chem 263: 18437-18442, 1988.

4. Young JC, Hoogenraad NJ and Hartl FU: Molecular chaperones Hsp90 and Hsp70 deliver preproteins to the mitochondrial import receptor Tom70. Cell 112: 41-50, 2003.

5. George R, Beddoe T, Landl K and Lithgow T: The yeast nascent polypeptide-associated complex initiates protein targeting to mitochondria in vivo. Proc Natl Acad Sci USA 95: 2296-2301, 1998.

6. Joza N, Pospisilik JA, Hangen E, et al: AIF: not just an apoptosisinducing factor. Ann NY Acad Sci 1171: 2-11, 2009.

7. Susin SA, Lorenzo HK, Zamzami N, et al: Molecular characterization of mitochondrial apoptosis-inducing factor. Nature 397: 441-446, 1999.

8. Otera H, Ohsakaya S, Nagaura Z, Ishihara N and Mihara K: Export of mitochondrial AIF in response to proapoptotic stimuli depends on processing at the intermembrane space. EMBO J 24: 1375-1386, 2005.

9. Yu SW, Wang H, Poitras MF, et al: Mediation of poly(ADPribose) polymerase-1-dependent cell death by apoptosis-inducing factor. Science 297: 259-263, 2002.

10. Fang HY, Chang CL, Hsu SH, et al: ATPase family AAA domain-containing $3 \mathrm{~A}$ is a novel anti-apoptotic factor in lung adenocarcinoma cells. J Cell Sci 123: 1171-1180, 2010.

11. Chiang YY, Chen SL, Hsiao YT, et al: Nuclear expression of dynamin-related protein 1 in lung adenocarcinomas. Mod Pathol 22: 1139-1150, 2009.

12. de Brito OM and Scorrano L: Mitofusin 2: a mitochondriashaping protein with signaling roles beyond fusion. Antioxid Redox Signal 10: 621-633, 2008.
13. de Brito OM and Scorrano L: Mitofusin 2 tethers endoplasmic reticulum to mitochondria. Nature 456: 605-610, 2008.

14. Pitts KR, Yoon Y, Krueger EW and McNiven MA: The dynaminlike protein DLP1 is essential for normal distribution and morphology of the endoplasmic reticulum and mitochondria in mammalian cells. Mol Biol Cell 10: 4403-4417, 1999.

15. Yoon Y, Pitts KR, Dahan S and McNiven MA: A novel dynaminlike protein associates with cytoplasmic vesicles and tubules of the endoplasmic reticulum in mammalian cells. J Cell Biol 140: 779-793, 1998.

16. Baixauli F, Martin-Cofreces NB, Morlino G, et al: The mitochondrial fission factor dynamin-related protein 1 modulates T-cell receptor signalling at the immune synapse. EMBO J 30: 1238-1250, 2011

17. Li Y, Sun Y, Hines JC and Ray DS: Identification of new kinetoplast DNA replication proteins in trypanosomatids based on predicted S-phase expression and mitochondrial targeting. Eukaryot Cell 6: 2303-2310, 2007.

18. Chow KC and Ross WE: Topoisomerase-specific drug sensitivity in relation to cell cycle progression. Mol Cell Biol 7: 3119-3123, 1987.

19. Shiao YJ, Lupo G and Vance JE: Evidence that phosphatidylserine is imported into mitochondria via a mitochondria-associated membrane and that the majority of mitochondrial phosphatidylethanolamine is derived from decarboxylation of phosphatidylserine. J Biol Chem 270: 11190-11198, 1995.

20. Csordas G, Renken C, Varnai P, et al: Structural and functional features and significance of the physical linkage between ER and mitochondria. J Cell Biol 174: 915-921, 2006.

21. Hayashi T, Rizzuto R, Hajnoczky G and Su TP: MAM: more than just a housekeeper. Trends Cell Biol 19: 81-88, 2009.

22. Vance JE: Phospholipid synthesis in a membrane fraction associated with mitochondria. J Biol Chem 265: 7248-7256, 1990.

23. de Kroon AI, Koorengevel MC, Vromans TA and de Kruijff B: Continuous equilibration of phosphatidylcholine and its precursors between endoplasmic reticulum and mitochondria in yeast. Mol Biol Cell 14: 2142-2150, 2003.

24. Neuspiel M, Schauss AC, Braschi E, et al: Cargo-selected transport from the mitochondria to peroxisomes is mediated by vesicular carriers. Curr Biol 18: 102-108, 2008.

25. Dhar SS, Ongwijitwat S and Wong-Riley MT: Nuclear respiratory factor 1 regulates all ten nuclear-encoded subunits of cytochrome c oxidase in neurons. J Biol Chem 283: 3120-3129, 2008.

26. Ongwijitwat $S$ and Wong-Riley MT: Is nuclear respiratory factor 2 a master transcriptional coordinator for all ten nuclearencoded cytochrome c oxidase subunits in neurons? Gene 360: 65-77, 2005.

27. Merkwirth $C$ and Langer T: Mitofusin 2 builds a bridge between ER and mitochondria. Cell 135: 1165-1167, 2008.

28. Lewin TM, Van Horn CG, Krisans SK and Coleman RA: Rat liver acyl-CoA synthetase 4 is a peripheral-membrane protein located in two distinct subcellular organelles, peroxisomes, and mitochondrial-associated membrane. Arch Biochem Biophys 404: 263-270, 2002.

29. Schrader M: Shared components of mitochondrial and peroxisomal division. Biochim Biophys Acta 1763: 531-541, 2006.

30. Chacinska A, van der Laan M, Mehnert CS, et al: Distinct forms of mitochondrial TOM-TIM supercomplexes define signal-dependent states of preprotein sorting. Mol Cell Biol 30: 307-318, 2010.

31. Bozidis P,Williamson CD and Colberg-Poley AM: Mitochondrial and secretory human cytomegalovirus UL37 proteins traffic into mitochondrion-associated membranes of human cells. J Virol 82: 2715-2726, 2008.

32. Burikhanov R, Zhao Y, Goswami A, Qiu S, Schwarze SR and Rangnekar VM: The tumor suppressor Par-4 activates an extrinsic pathway for apoptosis. Cell 138: 377-388, 2009.

33. Sun FC, Wei S, Li CW, Chang YS, Chao CC and Lai YK: Localization of GRP78 to mitochondria under the unfolded protein response. Biochem J 396: 31-39, 2006. 